\title{
The $X M M-\Omega$ project: gas mass fraction shape in high redshift clusters
}

\author{
R. Sadat ${ }^{1}$ and the $X M M-\Omega$ collaboration \\ ${ }^{1}$ Laboratoire d'astrophysique de l'observatoire Midi-Pyrénées, CNRS, UMR 5572, UPS, 14, \\ Av. E. Belin, 31400 Toulouse, France
}

\begin{abstract}
We present the study of the gas mass fraction, $f_{\text {gas }}$, properties of distant galaxy clusters observed within the $X M M-\Omega$ project, based on a sample of distant $(z \sim 0.5)$ clusters of median luminosity. We first study the $f_{\text {gas }}$ profiles traced up to the virial radius in both Einstein de Sitter (EdS) and concordance cosmologies. We find that the mean gas fraction profile of our high- $z$ sample is similar to the local mean profile and in good agreement with numerical simulation predictions. This result confirms the self-similarity of the shape predicted by the simple scenario of structure formation up to high redshifts. We observe $f_{\text {gas }}$ in the central part of distant clusters to lie below the local ones in the case of an EdS model, consistently with the observed evolution in the $L_{\mathrm{X}}-T$ relation, which is indeed found to be weaker than expected in a self-similar model. We investigate quantitatively this departure from scaling laws by comparing $f_{\text {gas }}$ in our distant $X M M$ clusters with those derived from the local sample at fixed scaled radii. We show that the internal structure is very complex, i.e. the variation of the gas mass fraction with radius, with temperature and with redshift reveals differences that cannot be described in a simple scaling scheme. The observed variations in the central parts are a clear indication that, in these regions, the argument based on the non-evolving $f_{\text {gas }}$ with redshift cannot be used to infer the cosmological parameters, given the present (lack of) understanding of the gas physics in clusters. At the virial radius, where this argument could be valid, we find that the $\Lambda$ CDM model is excluded at more than $3-\sigma$.
\end{abstract}

\section{Introduction}

Clusters of galaxies are unique cosmological probes whose statistical properties represent major sources of information for understanding the history of structure formation as well as for the determination of the cosmological parameters. In the simplest picture of purely gravitationally driven formation of virialized systems like galaxy clusters, it is expected that such objects exhibit self-similarity (Kaiser 1986). In this model physical properties of galaxy clusters obey scaling laws. The mass-temperature relation is consistent with the expected $M \propto T^{3 / 2}$ scaling while deviations from simple scaling predictions are observed in the $L_{\mathrm{X}}-T, M_{\text {gas }}-T$ relations for example. Moreover, self-similar models predict that the radial profile of any physical quantity should be similar independently of the cluster mass and redshift. Numerical simulations indicate that haloes of different masses follow a universal density profile, the so-called NFW profile (Navarro, Frenk \& White 1996). The X-ray emissivity profiles in hot galaxy clusters are also found to be very similar at least in the clusters outer parts (Ponman et al. 1999). More recently, it has been shown that $f_{\text {gas }}$ profiles are remarkably similar and seem to follow a universal shape (Roussel et al. 2000; hereafter RSB00). The self-similar model also makes predictions on the evolution of cluster properties. In particular the $L_{\mathrm{X}}-T$ relation should scale as $(z+1)^{3 / 2}$ in an Einstein de Sitter (EdS) universe. Several studies have found evidence of a weak evolution in the $L_{\mathrm{X}}-T$ relation (Sadat et al. 1998, Lumb et al. 2004, Vikhlinin et al. 2002). Using non-evolving physical properties of clusters as distance indicators can 
provide a new promising method for constraining cosmological parameters (Pen 1998, Sasaki 1996). The aim of the present study is to better understand the redshift evolution of the gas mass fraction scaling. We will discuss how the complexity of the internal structure can affect the application of the $f_{\text {gas }}$ test and will present our result based on eight high redshift clusters observed with $X M M$.

\section{The sample}

We base our analysis on the $X M M$ data obtained on a sample of eight distant SHARC clusters observed as part of the XMM- Rproject (Bartlett et al. 2002). This sample represents a homogeneous sample of eight bona fide clusters with median luminosities, between 2 and $15 \times 10^{44} \mathrm{erg} / \mathrm{s}$ (in the EdS cosmology with $h=0.5$ ) with redshifts in a relatively restricted range, between 0.45 and 0.65 . This sample is therefore expected to be fairly representative of the cluster population at high redshift. The detailed data reduction and analysis of this sample is presented in Lumb et al. (2004).

\section{The shape of $f_{\text {gas }}$ profiles}

We compute the gas mass fraction $f_{\text {gas }}=M_{\text {gas }} / M$ for each cluster of our high- $z$ sample at different radii $R_{\Delta}$ (the radius enclosing a density contrast $\Delta$ which depends on cosmology). We assume a $\beta$-model for the $\mathrm{X}$-ray gas distribution: the central electron density being computed from the Mekal constant. The total mass is computed from the $M-T$ relation calibrated from numerical simulations (Bryan and Norman, 1998). Inside a given radius $R_{\Delta}$, the binding mass is derived by assuming the so-called NFW profile. The temperature, $\beta$, the core radius, $r_{\mathrm{c}}$ and the Mekal constant are given in (Lumb et al. 2004). We estimate the apparent gas mass fraction within two different cosmological models (an $\Omega_{M}=1$ and a flat $\Lambda=0.7$ cosmology and $h=0.5$ ). The $f_{\text {gas }}$ radial distribution in the local sample is derived using the published gas mass measured up to the X-ray limiting radius $R_{\mathrm{X} \lim }(\mathrm{RSB} 00)$; upward this radius we use the gas masses computed at two radii $R_{1000}$ and $R_{2000}$ as published by Vikhlinin et al 1999 (hereafter VFJ99). For this comparison we do not correct for the clumping (Mathiesen et al. 1999), as the radial variation of this quantity is unknown. Furthermore, if the scaling actually holds, the emission of both local and distant clusters should be biased by this effect by the same amount, and therefore the lack of correction does not affect our comparison. In figure 1 we compare the $f_{\text {gas }}$ profile of the high- $z$ clusters sample with nearby clusters in both cosmologies.

While a noticeable difference on the amplitude appears depending on the cosmological model, the shape of the observed profiles of distant clusters are globally in good agreement with what has been inferred for clusters at low redshift: the apparent $f_{\text {gas }}$ profiles of our distant clusters increase from the center to the outer shells following the universal gas fraction shape. These measured $f_{\text {gas }}$ profiles still exibit a dispersion which is consistent with what has been previously found (RSB00). In the Einstein-de Sitter model the most central values of $f_{\text {gas }}$ appear to be smaller in distant clusters. Such deviation is consistent with the measured evolution of the $L_{\mathrm{X}}-T$ relation, weaker than expected if the scaling was actually strictly holding. Conversely, in the low density flat model, $f_{\text {gas }}$ values in the central parts of distant clusters seem to agree with the standard scaling, again in agreement with the evolution of the $L_{\mathrm{X}}-T$ relation in this cosmology. 

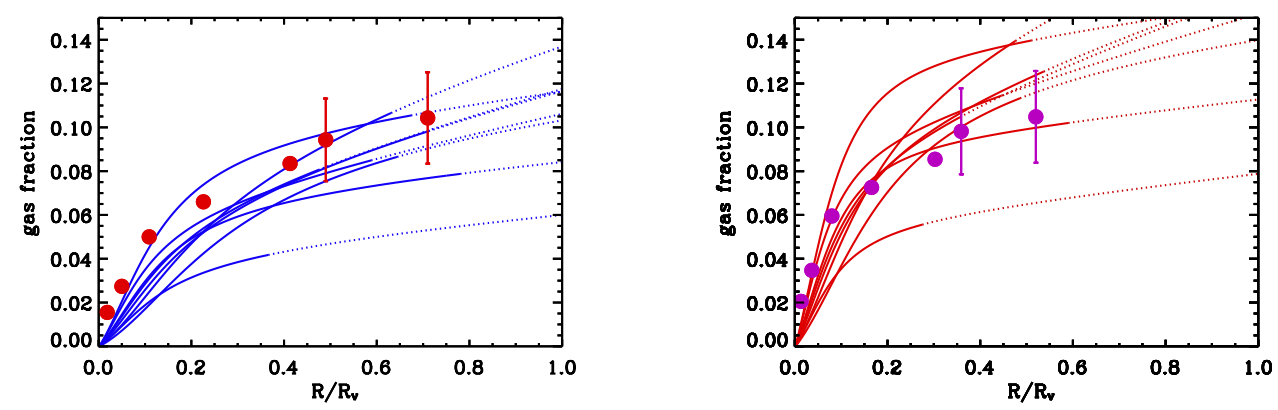

Figure 1. The scaled apparent $f_{\text {gas }}$ profiles of high- $z X M M$ clusters (solid lines) compared to the mean scaled profile of local clusters in EdS model (on the left) and in a $\Lambda$ CDM model (right side).

\section{1. $f_{\text {gas }}$ - Temperature correlation}

Before applying the test based on the assumption that $f_{\text {gas }}$ is universal and therefore does not vary with redshift we should keep in mind two possible sources of complexity: the gas mass fraction varies with radius (see fig. 1) and possibly with temperature. Such a trend has been observed from time to time on local samples. By examining the RSB00 sample used in Fig.1, we indeed found a clear trend of $f_{\text {gas }}$ to increase with temperature at least up to the X-ray detection limit $R_{\mathrm{X} \lim }$ (while this trend is much less clear on the global sample). However, this sample was not designed to offer a uniform sample for X-ray studies, therefore it has been used only as a guideline in the present study. For the following analysis, we will use the VFJ99 sample and compute $f_{\text {gas }}$ at their two fiducial radii $R_{2000}, R_{1000}$ as well at the virial radius. It should be noticed that distant clusters are expected to be on the bright tail of the distribution inducing a Malmquist bias and therefore a bias toward hotter temperature. If $f_{\text {gas }}$ actually varies with temperature this bias should be corrected for. In order to compare properly with our high- $z$ sample we need to compute $f_{\text {gas }}$ at the same radius in unit of the virial radius. In figure 2 we have plotted $f_{\text {gas }}$ at fixed radius $\left(R_{2000}, R_{1000}\right.$ and $\left.R_{\mathrm{v}}\right)$ in the local and high redshift clusters versus temperature for the two cosmological models. The local sample reveals a clear trend of the apparent $f_{\text {gas }}$ to increase with the temperature following $f_{\text {gas }} \propto T^{0.25}$. This trend is slightly more pronounced in the inner radius. The origin of this variation is unclear. It might either be an actual variaton of $f_{\text {gas }}$ in clusters, but it might also be due to a variation of the clumping of the gas with temperture. Whatever this variation is, it implies an evolution of the scaling. Comparing our $X M M$ clusters with local clusters at the virial radius where the $f_{\text {gas }}-T$ correlation is less pronouced shows that the gas mass fraction in the high- $z$ cluster sample matches fairly well the local sample in the case of an $\Omega_{M}=1$ model, while in a $\Lambda$ CDM model it is deviating from the gas fraction mean value of the local sample; this effect is more pronounced at $R_{1000}$ than at $R_{2000}$. Note that the gas mass fraction at the virial radius implies some extrapolation of the observed profile for clusters in our sample.

\section{Cosmological application}

The test based on the ansatz that the baryon (gas) fraction does not evolve with redshift has been used as a test for cosmological parameters determination (see Ettori et al. 2003 and Allen et al. 2002 for recent applications). From Fig. 2, at the virial radius, we can see that while the agreement between low and high- $z$ clusters is quite 

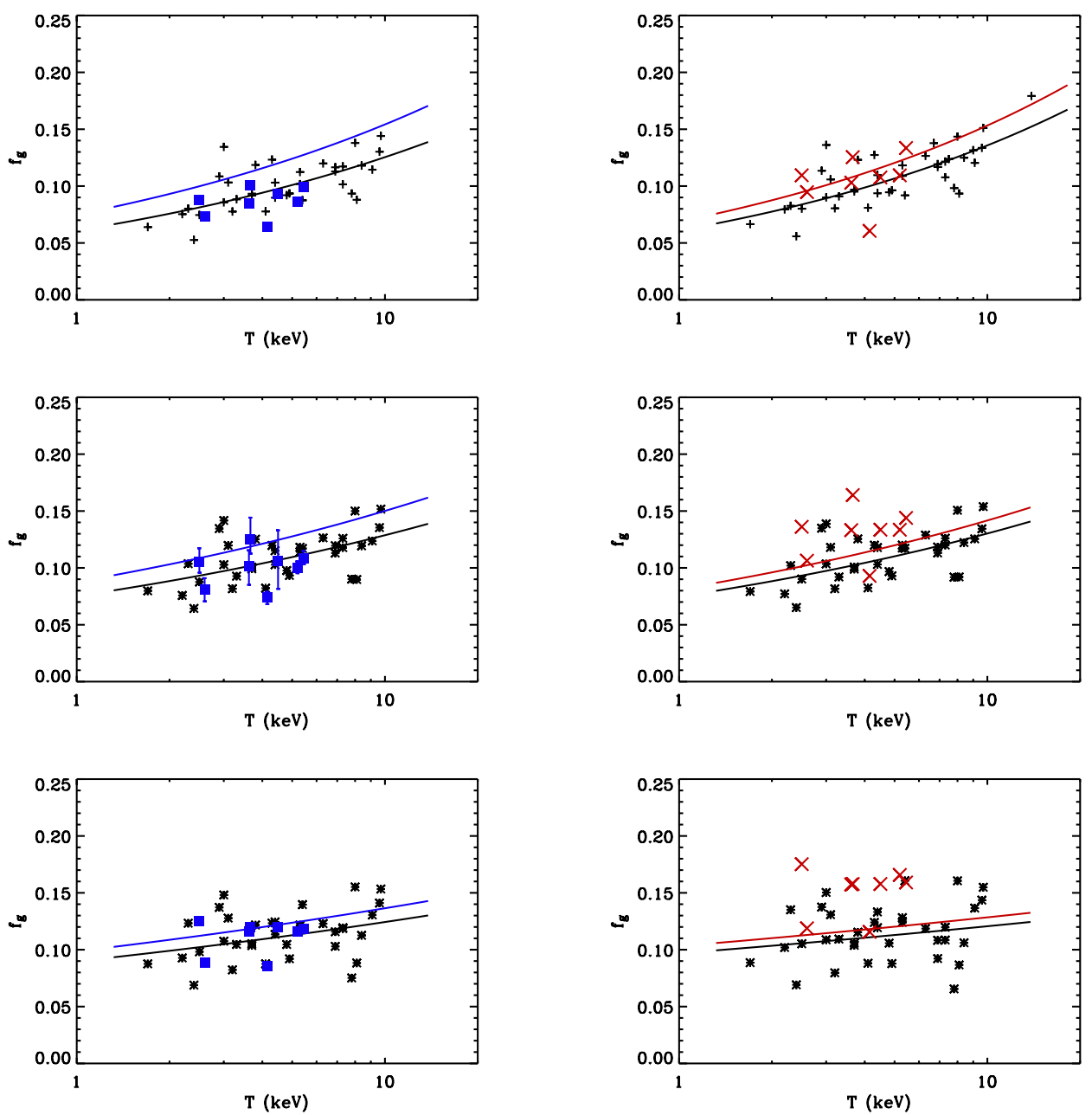

Figure 2. The apparent $f_{\text {gas }}$ versus temperature measured at three different radii $R_{2000}$ (top), $R_{1000}$ (middle) and $r_{\mathrm{v}}$ (bottom) in the outer part of the $X M M$ distant clusters compared to the same quantity evaluated at the same scaled radii in EdS model (on the left) and in a $\Lambda$ CDM model (right side). The black solid line is a fit to $f_{\text {gas }}$ of local clusters; we also plot as continuous colored (upper) line the predicted variation of $f_{\text {gas }}$ under the scaling assumption.

good in an EdS cosmology, there is a clear offset of $f_{\text {gas }}$ values computed in a $\Lambda$ CDM model for high-redshift clusters compared to local ones. Several aspects however make the direct cosmological interpretation difficult. First it is to notice that the clear trend with temperature could be a source of confusion: temperatures in our sample are fainter and therefore expected $f_{\text {gas }}$ are smaller. Comparing the gas fraction in our clusters with the hottest local clusters, would have led to favour the $\Lambda$ CDM model. The internal stucture of the gas seems not to be strictly identical in high and low redshift clusters, declining faster in the central part of high- $z$ clusters. This is consistent with what seems to emerge from Fig. 1. This complex internal structure could be the result of non gravitational heating of the gas which is currently advocated to explain the observed $L_{\mathrm{X}}-T$ relation. Possible systematic variations with radius, temperature and redshift, imply that the 
baryon fraction test should be performed with caution and as much as possible at the virial radius.

\section{Conclusion}

The observations of distant clusters with $X M M$ offer a unique possibility to investigate the outer emissivity of the gas distribution in distant X-ray clusters. We have shown how the observations of the distant SHARC clusters are leading to interesting results on this issue: the shape of the apparent $f_{\text {gas }}$ derived for those clusters is in rough agreement with the shape of the apparent $f_{\text {gas }}$ of local clusters. This is an independent confirmation that the scaled shape of the baryon fraction in clusters is in rough agreement with the shape found in numerical simulations (Frenk et al. 1999). By comparing our distant clusters to a sequence of local clusters, we found that a clear variation with temperature and redshift of the internal structure of clusters exists which cannot be described by some simple scaling relations. We use our high- $z$ sample to apply the cosmological test based on the assumption that $f_{\text {gas }}$ should have a universal value independent of redshift. At the virial radius we have found that the data are roughly consistent with the scaling hypothesis in the Einstein-de Sitter model, but not within the standard concordance model in which the observed gas mass fraction appears higher $(4 \sigma)$ than in clusters of the local universe. The internal structure of the gas presents however some complex variations that makes difficult a fully reliable use of the gas mass fraction for the determination of the cosmological parameters.

\section{References}

Allen, S. W.; Schmidt, R. W.; Fabian, A. C. 2002 MNRAS 334, L11-L15

Bartlett, J. et al. 2001, proceedings of the XXI rencontre de Moriond, astro-ph/0106098

Bryan, G.L. \& Norman, M.L. 1998 ApJ 495, 80-99

Ettori, S., Tozzi, \& P. Rosati 2003 MNRAS 305, 834

Frenk, C.S, White, S.D.M et al. 1999 ApJ 525, 554-582

Kaiser, N. 1986 MNRAS 222, 323-345

Lumb, D. et al. 2004, astro-ph/0311344, accepted for publication in $A \mathscr{E} A$

Mathiesen, B., Evrard, A.E. \& Mohr, J.J. 1999 ApJ 520, L21

Mohr, J.J., Mathiesen, B. \& Evrard, A.E. 1999 ApJ 517, 627-649

Navarro, J.F., Frenk, C.S., White, S.D.M. 1997 ApJ 490, 493-508

Pen, Ue-Li 1997 NewA 2, 309-317

Ponman, T.J., Cannon, D. B. \& Navarro, J. F. 1999 Nature 397, 135-137

Roussel, H., Sadat, R. \& Blanchard, A. $2000 \quad A \& A$ 361, 429-443

Sadat, R.\& Blanchard, A. $2001 \quad A \mathscr{E} A$ 329, 19-24

Sasaki, S. 1996 PASJ 48, 119-122

Vauclair, S.C. et al. $2003 \quad A \& A L 412,37-41$

Vikhlinin, A., Forman, W. \& Jones, C. 1999 ApJ 525, 47-57 